\title{
63. The Anatomical Origin of the Series Elastic Component in Cardiac Muscle
}

\author{
By Haruo Sugi and Akimitsu Kamiyama \\ Department of Physiology, School of Medicine, Teikyo University, \\ Itabashi-ku, Tokyo \\ (Communicated by Yasuji Katsuki, M. J. A., Dec. 12, 1977)
}

The two component model of Hill, ${ }^{1)}$ which consists of an elastic component in series with a contractile component, is widely applied to the muscle mechanics. In frog skeletal muscle, the extension of the series elastic component (SEC) with the maximum isometric force is about $1 \%$ of the resting length, ${ }^{2}$ and the force-extension curves of the SEC are scaled down in proportion to the isometric force, ${ }^{3), 4)}$ indicating that the SEC is not actually separate from the contractile component but may largely resides in the cross-bridges. In cardiac muscle, the origin of the SEC is more complex than in skeletal muscle; the extension of the SEC with the maximum isometric force in cardiac muscle is $5-10 \%$ of the initial length, ${ }^{5), 6)}$ being too large for the elastic extension of the cross-bridges. The present experiments were undertaken to give information about the source of the highly compliant SEC in cardiac muscle which remains to be determined. ${ }^{7), 8)}$

The right ventricular papillary muscles were rapidly removed together with the ventricular septa from dogs under pentobarbitol anesthesia. The ventricular septum was pinned securely to the side walls and the bottom of an experimental chamber, and the septal artery was cannulated ${ }^{9)}$ to perfuse the septum and the papillary muscle with oxygenated $\left(95 \% \mathrm{O}_{2}-5 \% \mathrm{CO}_{2}\right)$ Tyrode solution containing $(\mathrm{mM}): \mathrm{NaCl}, 147 ; \mathrm{KCl}, 2.7 ; \mathrm{CaCl}_{2}, 1.8 ; \mathrm{NaHCO}_{3}, 11 ; \mathrm{NaH}_{2} \mathrm{PO}_{4}$, 0.45 ; glucose, 5.6. The perfusate also contained defibrinized blood of sacrificed $\operatorname{dog}(1 \%)$. Perfusion was maintained at a rate of about $2 \mathrm{ml} / \mathrm{min}$. The distal tendon of the papillary muscle was tied to an L-shaped brass arm extending from an electromagnetic vibrator (Ling Dynamic Systems Ltd.) within $1 \mathrm{~mm}$ from the end of the muscle. The vibrator was driven with rectangular current pulses to produce rapid changes in muscle length. The force transducer consisted of a pair of semiconductor strain gauge glued to the front and rear surfaces of the vertical portion of the arm (resonance frequency, about $500 / \mathrm{sec}$ ), while the length transducer was a light beam-photodiode system. ${ }^{10)}$ To avoid complications arising from the parallel 
elastic component, ${ }^{11}$ the initial muscle length was set at a point where the resting tension was less than $5 \%$ of the maximum isometric force. The muscle was stimulated to contract with $5 \mathrm{msec}$ current pulses of above threshold through a pair of $\mathrm{Pt}$ wire electrodes. The papillary muscle-septum preparations could generate isometric force $(5-10 \mathrm{~g})$ definitely larger than that in the isolated papillary muscle preparations for many hours. At the peak of isometric force in response to stimulation, the muscle length was suddenly decreased to a variable extent (quick release), and the coincident reduction of force was observed on an oscilloscope (inset in Fig. 1). The experiments were made at room temperature $\left(24-26^{\circ} \mathrm{C}\right)$.

Fig. 1 shows the force-extension curve of the SEC obtained by the quick release method. The minimum amount of quick release required to reduce the peak force to zero, i.e. the extension of the SEC with the maximum isometric force, was 6-9\% of the initial muscle length $(11-15 \mathrm{~mm})$ in agreement with the values obtained in other studies.

To examine the anatomical origin of the SEC, the experiments were further performed, in which a number of fine carbon particles were firmly attached to the muscle surface, and the length changes

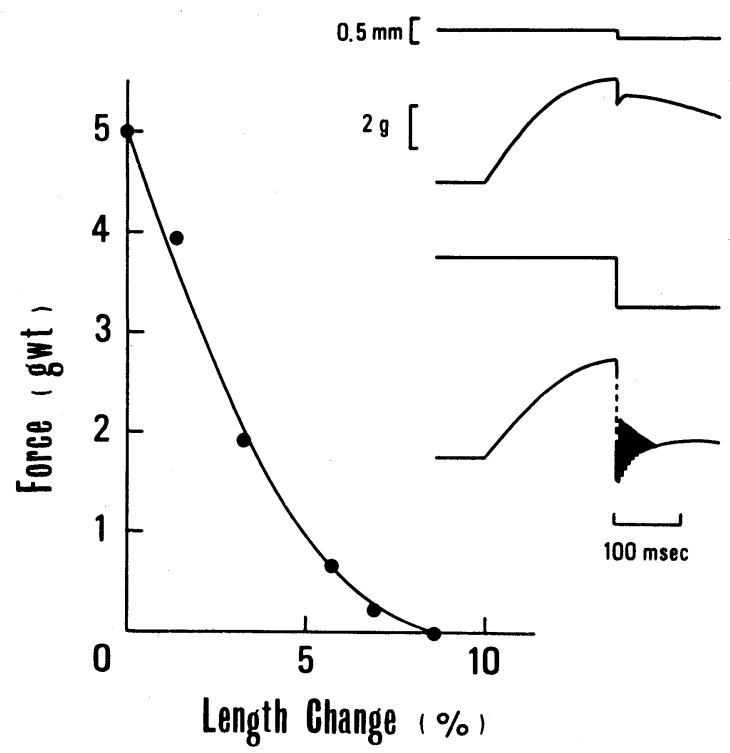

Fig. 1. Force-extension curve of the SEC in dog papillary muscle obtained by the quick release method. Forces immediately following the quick release are plotted against the amount of decrease in muscle length expressed in percentage of the initial length. Inset shows examples of length and force changes during a quick release. 

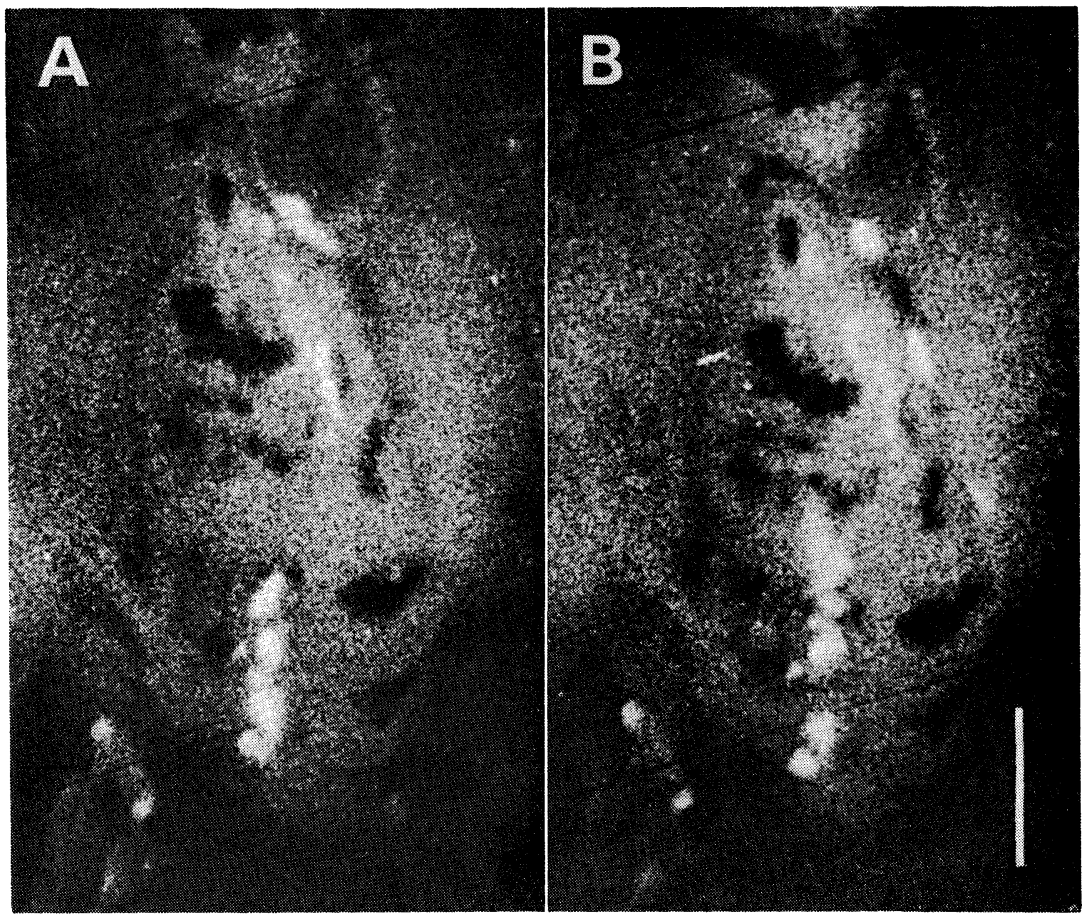

Fig. 2. Selected frames from a cinefilm of a papillary muscle at the peak of isometric force (A) and immediately after a quick relase (B) with which the force fell to zero. The distal tendon end of the muscle is located at the upper part of each frame. Each dark mass of carbon on the muscle consists of a number of fine carbon particles attached to the muscle surface. Calibration, $2.5 \mathrm{~mm}$.

of the muscle segments divided by the particles were recorded, by means of a $16 \mathrm{~mm}$ high-speed cine-camera (500 frames/sec, Hicam, Redlake Corp.) mounted on a Wild binocular microscope, during the development of isometric force and during the quick release (Fig. 2). To avoid the distortion of the muscle image due to the disturbance of the surrounding fluid caused by the release, the muscle was exposed to air and illuminated by a Nikon xenon lamp during the cinematographic recording. Fig. 3 illustrates a typical result of the length changes of the muscle segments during the development of isometric force (left) and during the quick release (right). The length of each muscle segment was not kept isometric during the development of isometric force; some segments shortened by stretching others, though the total muscle length remained almost unchanged. ${ }^{12}$ ) The local tissue movement disappeared when the isometric force reached around the peak, indicating that the forces exerted by all the segments balanced. 
During a quick decrease in muscle length (6-9\%), with which the peak tension was reduced to zero, the shortening was always observed in the distal and the proximal end segments and also in a segment at the middle part of the muscle, while no appreciable shortening was seen in the other muscle segments. It was also noticed that, during the development of isometric force, the above three segments were stretched by the other segments to an extent comparable to that of shortening during the quick release. These experiments could be repeated many times on one and the same preparation, producing reproducible results. In some experiments, the muscle was quickly stretched by about $10 \%$ at the peak of isometric force. The lengthening was also localized in the two end segments and a segment at the middle part of the muscle, no appreciable length change being observed in the other muscle segments.

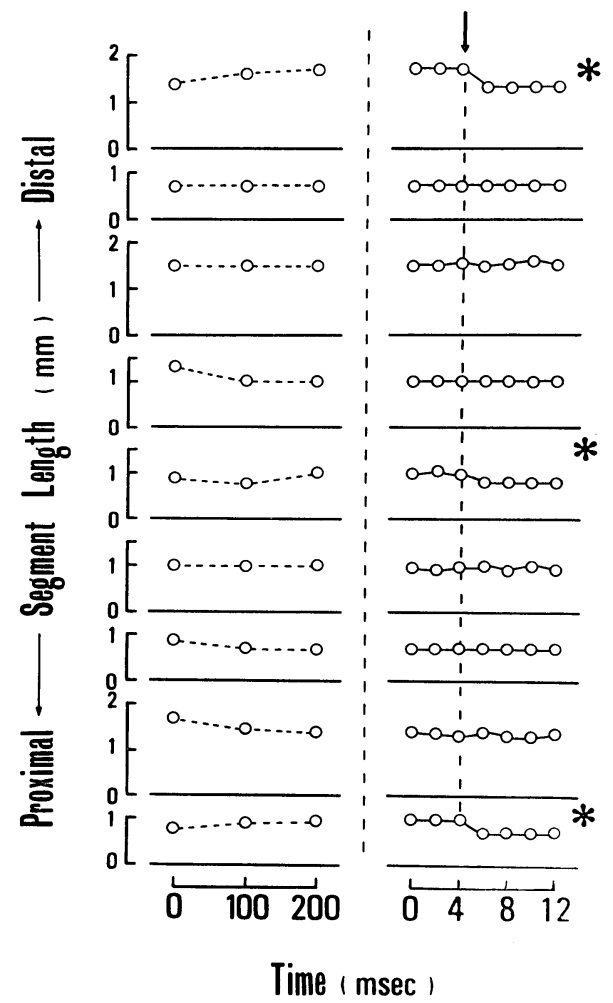

Fig. 3. Length changes of the muscle segments during the development of isometric force (left) and during a quick release (about 9\% of the initial muscle length) with which the force fell to zero (right). The quick release was made at the time indicated by arrow. Note that, during the quick release, the shortening is localized in the distal and the proximal end segments and also a segment in the middle part of the muscle. These three segments are indicated by asterisks. 
These results have demonstrated that, in dog papillary muscle, quick changes in muscle length are not uniformly distributed along the entire muscle length, but are mostly absorbed by the two end segments and also by a segment at the middle part. In the isolated papillary muscle, the source of the SEC is supposed to be an elastic strain of damaged tissue at both ends. ${ }^{12), 13)}$ In the present study, however, no tissue damage was produced at both ends of the papillary muscle-septum preparation. Thus, the localized length changes in our preparations may result from an inherent nature of complex network of component muscle cells. ${ }^{14)-16)}$ In conclusion, the present results strongly suggest that the highly compliant SEC in cardiac muscle may be mainly due to the localized elastic strain and recoil of the muscle tissue, which mask the contribution to the SEC of the crossbridge elasticity ${ }^{3,4)}$ or of the shearing through Z-lines and intercalated disks. ${ }^{17)}$

We wish to thank Miss Yoko Narikawa for her technical assistance.

\section{References}

1) Hill, A. V.: Proc. Roy. Soc. B, 126, 136-195 (1938).

2) Jewell, B. R., and Wilkie, D. R.: J. Physiol., 143 515-540 (1958).

3) Huxley, A. F., and Simmons, R. M.: Nature, 233, 533-538 (1971).

4) - : Cold Spring Harb. Symp. quant. Biol., 37, 669-680 (1972).

5) Sonnenblick, E. H.: Am. J. Physiol., 207, 1330-1338 (1964).

6) Bahler, A. S., Epstein, F., and Sonnenblick, E. H.: Ibid., 227, 794-800 (1974).

7) Noble, M. I. M., and Else, W.: Circ. Res., 31, 580-589 (1972).

8) Meiss, R. A., and Sonnenblick, E. H.: Am. J. Physiol., 226, 1370-1381 (1974).

9) Langer, G. A., and Brady, A. J.: J. gen. Physiol., 46, 703-719 (1963).

10) Sugi, H.: J. Physiol., 225, 237-253 (1972).

11) Brady, A. J.: J. Physiol., 184, 560-580 (1966).

12) Krueger, J. W., and Pollack, G. H.: Ibid., 251, 627-643 (1975).

13) Pollack, G. H., and Krueger, J. W.: Eur. J. Cardiol., 4 (Suppl.), 53-65 (1976).

14) Muir, A. W.: J. Anat., 99, 27-46 (1965).

15) Rodbard, S.: Anat. Rec., 166, 410 (1970).

16) Hort, W.: Arch. Pathol. Anat. Physiol., 333, 523-564 (1960).

17) Abbott, B. C., and Gordon, D. G.: Circ. Res., 36, 1-7 (1975). 